Pacific

Journal of

Mathematics

FREE GENUS ONE KNOTS WITH LARGE VOLUME

Mark Brittenham

Volume $201 \quad$ No. 1

November 2001 


\title{
FREE GENUS ONE KNOTS WITH LARGE VOLUME
}

\author{
MARK BRITTENHAM
}

\begin{abstract}
In this paper we construct families of hyperbolic knots with free genus one, whose complements have arbitrarily large volume. This implies that these knots have free genus one but arbitrarily large canonical genus.
\end{abstract}

\section{Introduction.}

A Seifert surface for a knot $K$ in the 3 -sphere is an embedded orientable surface $\Sigma$, whose boundary equals the knot $K$. In 1934, Seifert [Se] gave a very simple algorithm for constructing a Seifert surface for a knot, from a diagram, or projection, $D$ of the knot. Thus every knot has a Seifert surface.

Seifert's algorithm always builds a surface whose complement is a handlebody, something which is known as a free Seifert surface. The minimal genus among all free Seifert surfaces for $K$ is called the free genus $g_{f}(K)$ of $K$, while the minimal genus of a surface built by Seifert's algorithm applied to a projection of the knot $K$ is called the canonical genus $g_{c}(K)$ of $K$. (In keeping with this terminology, we will call a surface built by Seifert's algorithm canonical.) The above considerations immediately imply that, for any knot, $g_{f}(K) \leq g_{c}(K)$. It was shown by Kobayashi and Kobayashi $[\mathbf{K K}]$ that these numbers can be distinct; for $K$ the connected sum of $n$ copies of the double of a trefoil knot, $g_{f}(K)=2 n$ and $g_{c}(K)=3 n$.

An unusual feature of these examples is that the free genus minimizing surfaces are all compressible. We were interested in finding examples where the free and canonical genera differ, but the free genus minimizing surfaces were incompressible. In doing so, we were led in a natural way to consider hyperbolic knots, in order to exploit a relationship between canonical genus and volume.

In a previous paper $[\mathbf{B r}]$ we showed that hyperbolic knots with bounded canonical genus have complements with bounded volume. The bound on volume can in fact be chose to be linear in the canonical genus. In this paper we show, by contrast, that there is no corresponding bound in terms of the free genus of the knot.

Theorem. There exist hyperbolic knots with free genus one and arbitrarily large hyperbolic volume. 
These two results together show that there are free genus one hyperbolic knots with arbitrarily large canonical genus. Since a free genus one Seifert surface for a nontrivial knot is always incompressible, the knots we build also provide examples of knots with incompressible free Seifert surfaces which cannot be built by applying Seifert's algorithm to a projection of the knot. We should also note that a knot with free genus one has genus one, and so we immediately obtain the following corollary.

Corollary. There exist hyperbolic knots with genus one and arbitrarily large hyperbolic volume.

\section{Building free genus one knots.}

It is easy to build knots with free genus (at most) one, simply by building a genus one surface whose spine is an unknotted graph in the 3 -sphere. The complement of the surface is homeomorphic to the complement of the graph, and so is a handlebody. Consequently, the boundary of the surface has a genus 1 free Seifert surface, and so has free genus at most one. The best examples of this (and the starting point for our examples) are the 2-bridge knots (Figure 1) corresponding to the continued fractions $[2 \mathrm{u}, 2 \mathrm{v}]$ (see $[\mathbf{H T}]$ for notation). These surfaces are in fact isotopic to canonical surfaces for different projections of these knots.

This gives an infinite family of free genus one knots. However, since all of these knots can be built by doing $1 / u$ (and $1 / v$ ) Dehn surgeries on two of the unknotted components of the Borromean rings (Figure 1), these knots have hyperbolic volume smaller than the hyperbolic volume of the link [Th1], and so have bounded volume.

In order to insure that our free genus one knots will have large volume, we will rely on a result of Adams [Ad], which states that a complete hyperbolic manifold with $r$ cusps must have volume at least $r V_{0}$, where $V_{0}$ is the volume of a regular ideal tetrahedron. We will therefore build our knots by doing $1 / n_{i}$ Dehn surgeries on the (unknotted) components of a hyperbolic link with $r$ components. By a result of Thurston [Th1], for large values of the $n_{i}$, the resulting knots will also be hyperbolic and have volume close to that of the link. What we shall see is that for the particular link we choose, the resulting knots can also be seen to have free genus one Seifert surfaces, and so have free genus (at most) one.

The basic idea is to take one of the knots $K$ in Figure 1 and throw an extra loop $K_{1}$ around the 'waist' of the Seifert surface $F$; see Figure 2 . This loop $K_{1}$ lies on a 2-sphere $S$ bounding a 3-ball $B ; B \cap F$ is a disk and $S \cap F$ consists of four arcs. If we look at $M_{0}=B \backslash \operatorname{int}(N(F))$, it is a genus four handlebody which has the structure of a sutured manifold, where the sutures are the four loops $S \cap \partial(N(F))$. There are four obvious product disks for this sutured manifold (sitting in the plane of the paper, in the figure), so that, 


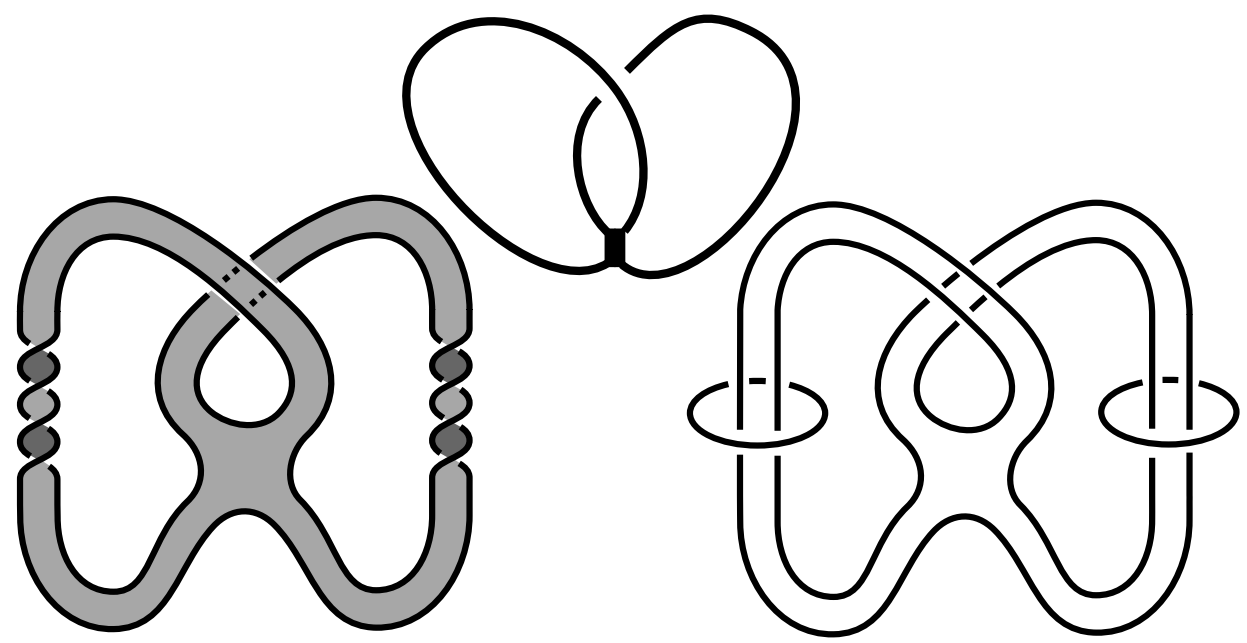

Figure 1.
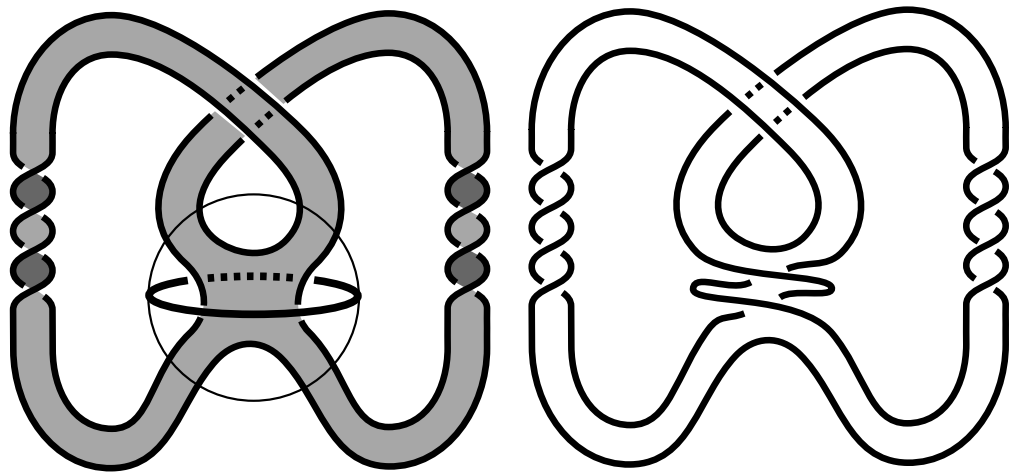

Figure 2.

as a sutured manifold, $M_{0}$ is a product sutured manifold (four-punctured sphere) $\times I$. In particular, the four-punctured sphere $B \cap \partial N(F)$ is isotopic, in $M_{0}$, to $S \backslash \operatorname{int}(N(F))$.

If we now do $1 / n$ Dehn surgery on $K_{1}$, then since $K_{1}$ is unknotted, the resulting manifold will be a 3 -sphere, and so the image of $K$ will be a knot $K^{\prime}$ in the 3 -sphere. By Kirby calculus (see [Ro]), $K^{\prime}$ can be obtained from $K$ by cutting $K$ along a disk $D$ spanning $K_{1}$, giving the resulting strands $n$ right-handed twists, and regluing. Similarly, the Seifert surface for $K$ gives a Seifert surface $F^{\prime}$ for $K^{\prime}$, by cutting, twisting, and regluing.

However, from the point of view of the ball $B$ (or, more precisely, a ball slightly smaller than $B$ ), nothing has really happened; we have cut 


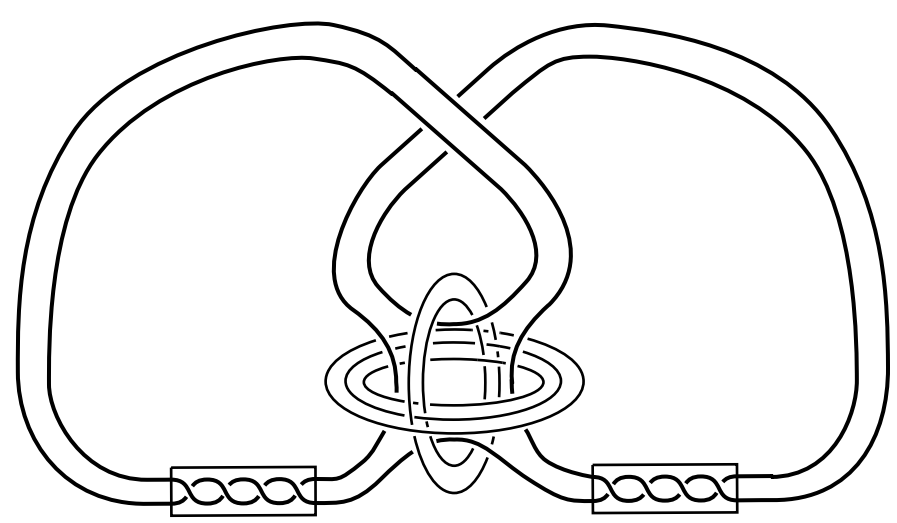

Figure 3.

the ball open along a disk, given one half of it $n$ full twists, and reglued by the identity map. Consequently, $B \backslash \operatorname{int}\left(N\left(F^{\prime}\right)\right) \cong B \backslash \operatorname{int}(N(F))$ is also a product sutured manifold, and so $B \cap \partial N\left(F^{\prime}\right)$ is isotopic, in $B \backslash \operatorname{int}\left(N\left(F^{\prime}\right)\right)$, to $S \backslash \operatorname{int}\left(N\left(F^{\prime}\right)\right)=S \backslash \operatorname{int}(N(F))$. Therefore, $S^{3} \backslash \operatorname{int}\left(N\left(F^{\prime}\right)\right) \cong S^{3} \backslash(\operatorname{int}(B \cup$ $\left.\left.N\left(F^{\prime}\right)\right)\right)=S^{3} \backslash(\operatorname{int}(B \cup N(F))) \cong S^{3} \backslash \operatorname{int}(N(F))$ is also a handlebody. $F^{\prime}$ is therefore a free genus one Seifert surface for $K^{\prime}$. Note that we could in fact have chosen any loop on $S$ to base this construction on; it would still bound a disk in the 3 -ball $B$, and so the argument above would go through without change. A more complicated loop would, however, unnecessarily complicate our arguments below.

More generally, we can throw many extra loops $K_{i}$ around $F$, on concentric 2-spheres, alternating which direction around the waist of $F$ we go (Figure 3), and do $1 / n_{i}$ Dehn surgeries on each of them. Since without $K$ these loops would together form a trivial link - they lie on disjoint 2-spheres - the resulting manifold will again be the 3 -sphere, and so $K$ will be taken to a new knot $K^{\prime}$ in the 3 -sphere. By working inductively out from the centermost 2 -sphere, cutting along disks, twisting, and regluing, we can also see that our Seifert surface $F$ will be taken to a free Seifert surface for $K^{\prime}$; at each step the argument is identical to the one given above. We therefore can produce knots with free genus at most one by this iterative construction, as well.

What we do not yet know is that these knots $K^{\prime}$ are hyperbolic, or that they have large volume. What we will show, however, is that the links $L_{n}$ $=K \cup K_{1} \cup \ldots K_{n}$ are all hyperbolic. By the result of Adams, for large $n$ they therefore have large volume, and so when the $n_{i}$ are all large, $K^{\prime}$ will have large volume (and, in particular, is therefore nontrivial, hence has free genus exactly one). This will complete the proof of our theorem. 


\section{The links $L_{n}$ are hyperbolic: Preliminaries.}

We now demonstrate that the links $L_{n}$ have hyperbolic complement; that is, the compact manifolds $M_{n}=X\left(L_{n}\right)=S^{3} \backslash \operatorname{int}\left(N\left(L_{n}\right)\right)$ have hyperbolic interior of finite volume. By Thurston's Geometrization Theorem [Th2], we must show that

(1) $X\left(L_{n}\right)$ is irreducible,

(2) $X\left(L_{n}\right)$ is $\partial$-irreducible, i.e., $\partial X\left(L_{n}\right)$ is incompressible in $X\left(L_{n}\right)$,

(3) $X\left(L_{n}\right)$ is atoroidal, i.e., an incompressible torus $T$ in $X\left(L_{n}\right)$ is parallel to $\partial X\left(L_{n}\right)$, and

(4) $X\left(L_{n}\right)$ is anannular, i.e, any properly embedded incompressible annulus $A$ in $X\left(L_{n}\right)$ is $\partial$-parallel.

In this section we will set up a few additional assumptions and prove some preliminary results, which will allow us to develop the machinery to prove these assertions. The basic idea is that, since this is a proof by construction, we can (and will) make whatever assumptions we feel are necessary to bring a wide array of different tools to bear on the problem, from standard cut and paste arguments to homological intersection numbers to normal forms for words in a free group.

The main assumption we will need to make, in order to prove that the links $L_{n}$ are hyperbolic, is that our underlying 2-bridge knot $K$, given by the continued fraction $[2 u, 2 v]$, lying at the center of the links $L_{n}$ has $|u|,|v| \geq 2$. Experimental evidence (finding hyperbolic structures using SnapPea [We]) suggests that in fact the links are always hyperbolic, without this added assumption, but some of our arguments will not go through in greater generality. We will also assume that $n \geq 3$, since this will, in the end, make the verification of condition (4) almost immediate. Again, experimental evidence suggests that this is not a necessary assumption.

Central to our proof that the knots obtained by $1 / n_{i}$ surgeries have free genus at most one was that the Seifert surface $F$ for the knot $K$ is disjoint from all of the added components $K_{i}$. In fact, each loop $K_{i}$ bounds a disk $D_{i}$ in $S^{3}$ which meets $F$ in an arc $\alpha_{i}$ (Figure 4). We will assume that we have pushed these disks slightly off of one another, so that if $i-j$ is even, then $D_{i}$ and $D_{j}$ are disjoint. When $i-j$ is odd and $j<i$, then $D_{j} \cap D_{i}$ consists of an $\operatorname{arc} \beta_{j i}$ contained in the interior of $D_{i}$. In particular, $D_{j} \cap K_{i}$ is empty, and $D_{i} \cap K_{j}$ consists of two points. Also, for each $i, D_{i} \cap\left(F \cup D_{1} \cup \ldots \cup D_{i-1}\right)$ is a finite tree, consisting of parallel arcs $\beta_{j i}$ each pierced by the arc $\alpha_{i}$ exactly once (see Figure 4). The surface $F$ is two-sided; we will arbitrarily assign it a normal orientation, and call one side of the surface $F_{+}$and the other side $F_{-}$. (Formally, we should think of this as being the two sides of $\partial N(F)$ in $X\left(L_{n}\right)$, but we won't really make such a distinction.)

An important point to notice is that not only is $S^{3} \backslash F$ a handlebody (of genus 2), since $F$ is a free Seifert surface, but $S^{3} \backslash\left(F \cup D_{i} \cup \ldots \cup D_{n}\right)$ is 


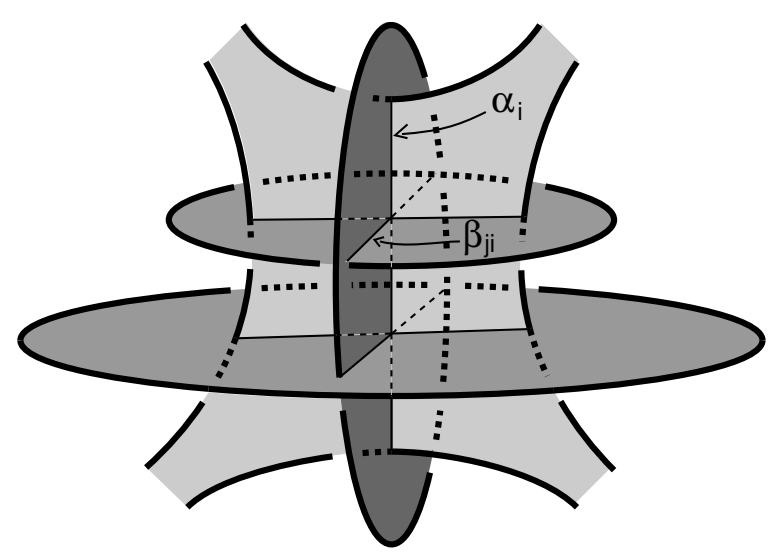

Figure 4.

a handlebody (of genus 2), as well. This is because $A_{i}=D_{i} \backslash \operatorname{int} N\left(F \cup D_{1} \cup\right.$ $\left.\ldots \cup D_{i-1}\right)$ is an annulus. Therefore, $X_{i}=S^{3} \backslash \operatorname{int} N\left(F \cup D_{1} \cup \ldots \cup D_{i}\right)$ is homeomorphic to $X_{i-1}=S^{3} \backslash \operatorname{int} N\left(F \cup D_{1} \cup \ldots \cup D_{i-1}\right) ; X_{i-1} \backslash X_{i}$ is a solid torus neighborhood of $A_{i}$, so $X_{i-1}$ is obtained from $X_{i}$ by gluing this solid torus to $\partial X_{i}$, along an annulus. Pushing $\partial X_{i}$ to $\partial X_{i-1}$ through this solid torus gives an isotopy in $X_{i-1}$ from $X_{i}$ to $X_{i-1}$.

This fact will allow us to take an inductive approach to our proof of property (3). We will start with an alleged essential torus $T$, and argue that we can find a (possibly different) torus disjoint first from $F$, and then, inductively, from each of the disks $D_{i}$. After we are done we will have an essential torus disjoint from all of them, which therefore sits in a handlebody. But since a handlebody is atoroidal, this will give us our contradiction.

In the rest of this section we collect together several lemmas which will tell us that certain kinds of intersections of a torus with $F$ and with the disks $D_{i}$ are not possible.

Lemma 1. For every $i$, there is no essential embedded annulus $A$ in $S^{3} \backslash \operatorname{int} N\left(F \cup K_{i}\right)$ with one $\partial$-component on $F_{ \pm}$and the other $\partial$-component on $\partial N\left(K_{i}\right)$.

Proof. This is easiest to see using a slightly different picture of $F$ (see Figure 5). $\pi_{1}\left(F_{+}\right), \pi_{1}\left(F_{-}\right)$, and $\pi_{1}\left(S^{3} \backslash \operatorname{int} N(F)\right)=\pi_{1}(H)$ are all free groups of rank two; using one of the bases depicted in Figure 5b (depending upon which of $F_{+}, F_{-}$we work with), we can see that, as subgroups of $\pi_{1}(H)=F(a, b), \pi_{1}\left(F_{+}\right)$is generated by $a^{u}$ and $a b^{v}$, and $\pi_{1}\left(F_{-}\right)$is generated by $b^{v}$ and $b a^{u}$. On the other hand, $K_{i}$ can be represented by either $a b$ or $a b^{-1}$, depending on the parity of $i$.

From the point of view of homotopy theory, an annulus described in the statement of the lemma gives a free homotopy from a loop representing a 


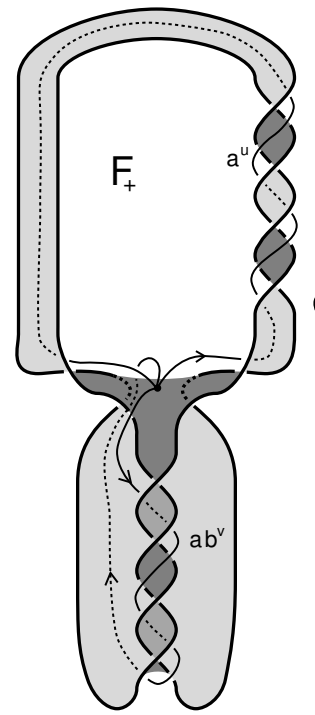

(a)

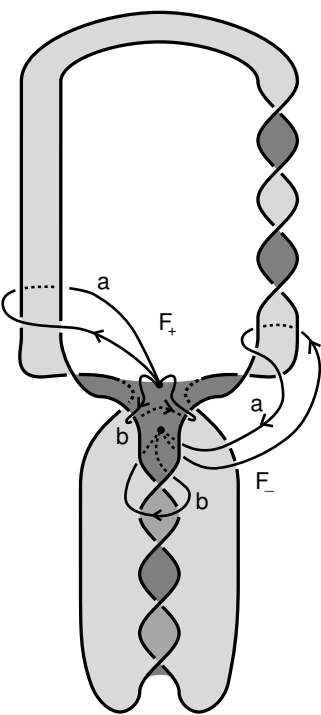

(b)

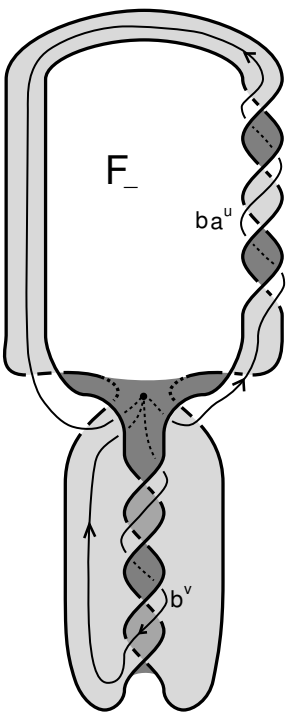

(c)

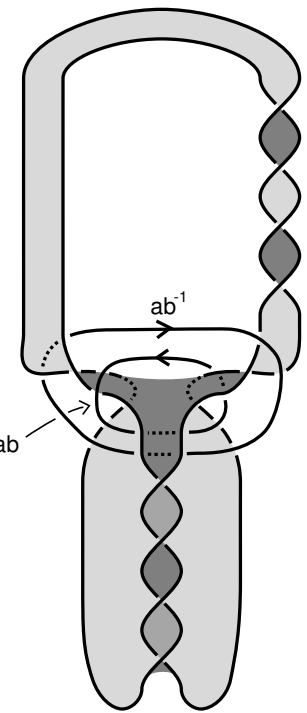

(d)

Figure 5 .

power of $a b^{ \pm 1}$ to a loop representing an element of $\pi_{1}\left(F_{ \pm}\right)$, and so from the point of view of fundamental groups, $\left(a b^{ \pm 1}\right)^{n}$, for some $n$, is conjugate in $F(a, b)$ to a word in the subgroup generated by $\left\{a^{u}, a b^{v}\right\}$ or $\left\{a^{u} b^{-1}, b^{v}\right\}$.

Conjugation preserves exponent sums in a free group, and so in the first case the exponent sum for $b$ will be $\pm n=k v$, so $v$ divides $n$, while in the second case the exponent sum for $a$ will be $n=k^{\prime} u$, so $u$ divides $n$. Since by our earlier hypothesis both $|u|$ and $|v|$ are greater than 2 , we have $|n| \geq 2$, or $n=0$. (This is essentially the only place in our proofs where these hypotheses on $u$ and $v$ will be used.)

But $n=0$ implies that $A$ meets $\partial N\left(K_{i}\right)$ in a meridian loop (since the boundary is embedded), and so, capping $A$ off with a meridian disk produces a disk $D$ with boundary on $F_{ \pm}$, meeting $K_{i}$ in a single point. Since $F_{ \pm}$is incompressible, $\partial D$ bounds a disk in $F$ which, together with $D$ forms a 2sphere in $S^{3}$ meeting $K_{i}$ in a single point, a contradiction (since a 2-sphere separates $S^{3}$ ). Therefore, $|n| \geq 2$.

A word in the free group $F(a, b)$ is said to be in normal form [MKS] if the letters $a$ and $a^{-1}$, and the letters $b$ and $b^{-1}$, do not occur side by side. Every element of the free group $F(a, b)$ has a unique normal form, which can be obtained by starting with a word representing the element and continually cancelling such adjacent pairs. 
But the word $x\left(a b^{ \pm 1}\right)^{n} x^{-1}$, when put into normal form, must, since $|n| \geq$ 2 , contain one of the strings

$$
\begin{gathered}
a b a b, \quad b a b a, a b^{-1} a b^{-1}, b^{-1} a b^{-1} a, \\
b^{-1} a^{-1} b^{-1} a^{-1}, a^{-1} b^{-1} a^{-1} b^{-1}, b a^{-1} b a^{-1}, \text { or } a^{-1} b a^{-1} b .
\end{gathered}
$$

For example, for $x(a b)^{n} x^{-1}$ with $n \geq 2$, one of the first two strings must appear. This can be proved by induction on the length of the normal word representing $x$. If we assume $x$ is written in normal form, the only way the initial string $a b a b$ of the center word, or the final $a b a b$, can be altered as we shorten our word to normal form is if $x$ ends in $a^{-1}$, or $x^{-1}$ begins with $b^{-1}$. But then either $x^{-1}$ begins with $a$ or $x$ ends with $b$, and so we can write

$$
\begin{gathered}
x(a b)^{n} x^{-1}=y a^{-1}(a b)^{n} a y^{-1}=y(b a)^{n} y^{-1} \quad \text { or } \\
x(a b)^{n} x^{-1}=\left(z^{-1} b\right)(a b)^{n}\left(b^{-1} z\right)=z^{-1}(b a)^{n} z .
\end{gathered}
$$

Then by induction (since the word length of the conjugating element has decreased), we are done; the base case $x=1$ is obvious. The fact that in the inductive step $a b$ became $b a$ is not a problem, since our conclusion is symmetric in $a$ and $b$; we simply imagine making our initial statement symmetric in $a$ and $b$ as well. The other pairs of possibilities listed above occur for the other combinations of exponent of $b$ and sign of $n$. Consequently, the normal form for our word $x\left(a b^{ \pm 1}\right)^{n} x^{-1}$ contains both an $a^{ \pm 1}$ surrounded on both sides by $b$ 's, and a $b^{ \pm 1}$ surrounded on both sides by $a$ 's.

This word is, by our argument above, contained in one of the (free) subgroups generated by $\left\{a^{u}, a b^{v}\right\}$ or $\left\{b a^{u}, b^{v}\right\}$. But this is impossible, because $u$ and $v$ are both at least 2 . In the first case, every occurrence of the letter $b$ will come in the form $\left(b^{v}\right)^{k}$, since $a^{u}$ contains no $b$ 's, and so it is impossible to have a single $b$ surrounded by $a$ 's in the normal form for the word. In the second case, every occurrence of the letter $a$ will come in the form $\left(a^{u}\right)^{k}$, since $b^{v}$ contains no $a$ 's, and so it is impossible to have a single $a$ surrounded by $b$ 's in the normal form for the word. Consequently, there can be no annulus running from $\partial N\left(K_{i}\right)$ to $F_{ \pm}$.

Lemma 2. For every $i \neq j$, there is no essential annulus properly embedded in $X\left(L_{n}\right)$ with one $\partial$-component on $\partial N\left(K_{i}\right)$ and the other $\partial$-component on $\partial N\left(K_{j}\right)$.

Proof. Suppose we have such an annulus $A$; consider $A \cap F \subseteq A$. Since $F \cap \partial A=\emptyset$ and $A \cap \partial F=\emptyset$, this intersection consists of loops. Since $F$ is incompressible, any loops which are trivial in $A$ can be removed by disk swapping. Any remaining loops must all be parallel to $\partial A$; if there are any, then an outermost such loop cuts off an annulus from $A$ with one $\partial$-component on $\partial N\left(K_{i}\right)$ or $\partial N\left(K_{j}\right)$ and the other $\partial$-component on $F_{ \pm}$, contradicting Lemma 1. So $A \cap F=\emptyset$. There are now two cases to consider: 


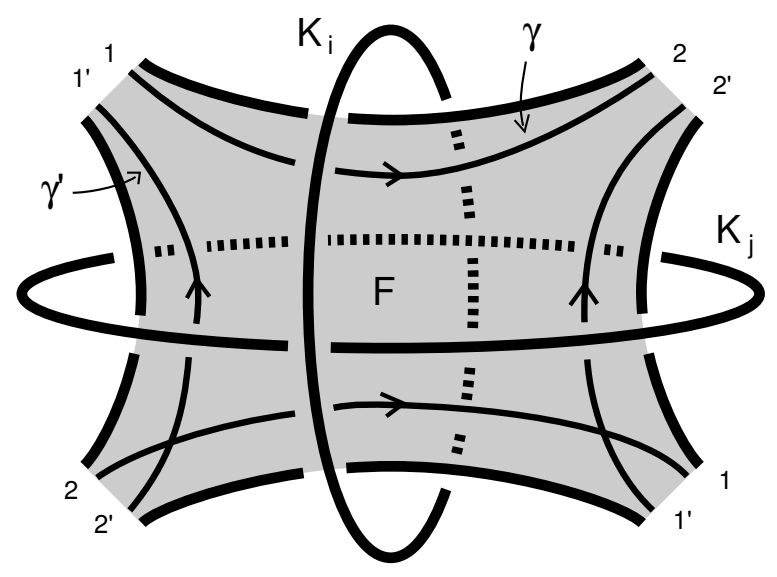

Figure 6.

Case 1: $i-j$ is odd.

In this case, $F, K_{i}$, and $K_{j}$ are as in Figure 6. There is a loop $\gamma$ in $F$ which has homological linking numbers (in $S^{3}$ ) 2 with $K_{i}$ and 0 with $K_{j}$. $\partial A \cap \partial N\left(K_{i}\right)$ is a curve of some slope $a_{i} / b_{i}$ on $\partial N\left(K_{i}\right)$, and $\partial A \cap \partial N\left(K_{j}\right)$ is a curve of slope $a_{j} / b_{j}$ on $\partial N\left(K_{j}\right)$. Since $A \cap F=\emptyset$, we have $A \cap \gamma=\emptyset$, and so $A$ represents a homology in the complement of $\gamma$ between its two boundary curves. These boundary curves represent the homology classes $b_{i}\left[K_{i}\right]=2 b_{i}$ and $b_{j}\left[K_{j}\right]=0 b_{j}=0$ in $H_{1}\left(S^{3} \backslash \gamma\right)$, so $b_{i}=0$. Similarly, a curve $\gamma^{\prime}$ can be found with the appropriate linking numbers, showing that $b_{j}=0$. This implies that both $\partial$-components of $A$ are meridian loops; capping off with meridian disks gives us a 2 -sphere in $S^{3}$ meeting each of the loops $K_{i}, K_{j}$ exactly once, a contradiction. Therefore, the annulus $A$ cannot exist.

Case 2: $i-j$ is even.

We may assume $j<i$, and so, setting $k=i-1, j<k<i$. We then have a situation like in Figure 7. Consider $A \cap D_{j} \subseteq D_{j}$. There is an arc of $F \cap D_{j}=\alpha_{j}$ between the two points of $K \cap D_{j}$, and since $A$ misses $F$, it misses $\alpha_{j} . A \cap D_{j}$ therefore consists of trivial arcs, trivial loops, and loops surrounding the arc $\alpha_{j}$. Innermost trivial loops can be removed by disk swapping with the corresponding disk in $A$, and outermost trivial arcs can be removed since $A$ is $\partial$-incompressible. Note that this implies that $A \cap \partial N\left(K_{j}\right)$ misses $\partial D_{j}$, and so represents a longitude of $\partial N\left(K_{j}\right)$.

This leaves loops travelling around $\alpha_{j}$; they must all be parallel to $\partial D_{j}=$ $K_{j}$, and therefore all parallel to one another. These loops must be nontrivial on $A$, since otherwise we can use the disk bounded by an innermost trivial loop on $A$, together with the annulus in $D_{j}$ that the loop cuts off to build a 


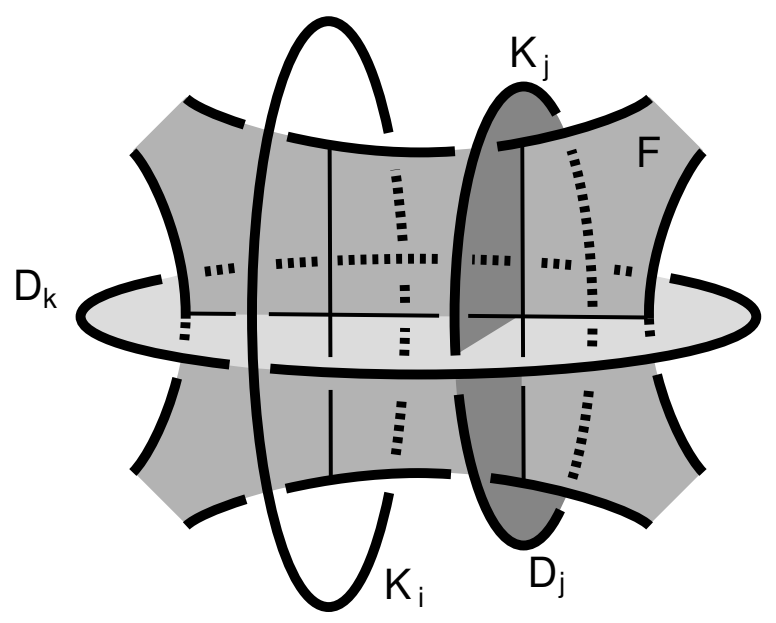

Figure 7.

disk $D^{\prime}$ disjoint from $F$ with $\partial D^{\prime}=K_{j}$. But this contradicts the fact that $K_{j}$ has homological intersection number 2 with one of the two loops $\gamma, \gamma^{\prime}$ from Figure 6. Then we can use the loop in $A$ closest to the $\partial$-component on $\partial N\left(K_{i}\right)$, cutting off an annulus from $A$, together with the annulus in $D_{j}$ that it cuts off, to build a new annulus $A^{\prime}$ between $\partial N\left(K_{i}\right)$ and $\partial N\left(K_{j}\right)$. Since both boundary components are essential, and lie on distinct $\partial$-tori, $A^{\prime}$ is essential. We can then push this annulus off of $D_{j}$ to make them disjoint. Setting $A=A^{\prime}$, we can therefore assume that $A \cap D_{j}=\emptyset$.

Technically, this new annulus might hit some of the loops $K_{r}$ for $r<j$; what we will actually show, therefore, is that there can be no essential annulus in $X\left(K \cup K_{j} \cup K_{k} \cup K_{i}\right)=X$. This will suffice, since our original annulus $A$ would be essential in this manifold, as well; any compressing disk for $A$ in $X$ could be pushed off of $F$ by disk swapping, since $A \cap F=\emptyset$, giving us a disk $D^{\prime}$ as in the previous paragraph, a contradiction. Since $A$ has its $\partial$-components on distinct $\partial$-tori, there can be no $\partial$-compressing disks, either.

Now consider $A \cap D_{k}$ (Figure 8). $A$ is disjoint from $\partial D_{k}=K_{k}$, and since $K_{j}$ meets $D_{k}$ in a pair of points, and $A \cap \partial N\left(K_{j}\right)$ is a longitude, $A \cap D_{k}$ consists of circles plus a single arc $\alpha$ joining the two points of $K_{j} \cap D_{k}$. Thinking of this arc as being in the annulus $D_{k} \backslash \operatorname{int} N\left(F \cup D_{j}\right)$, it is $\partial$-parallel, and so simply goes to the right or left around the tree $D_{j} \cap\left(F \cup D_{j}\right)$, say right.

But in $A, \alpha$ is $\partial$-parallel, since it joins a component of $\partial A$ to itself, and so cuts off a disk $\Delta$ from $A$, which is therefore disjoint from $F$ (since $A$ is). $\Delta \cap \partial N\left(K_{j}\right)$ is an arc of a longitude, running above or below the disk $D_{k}$, 


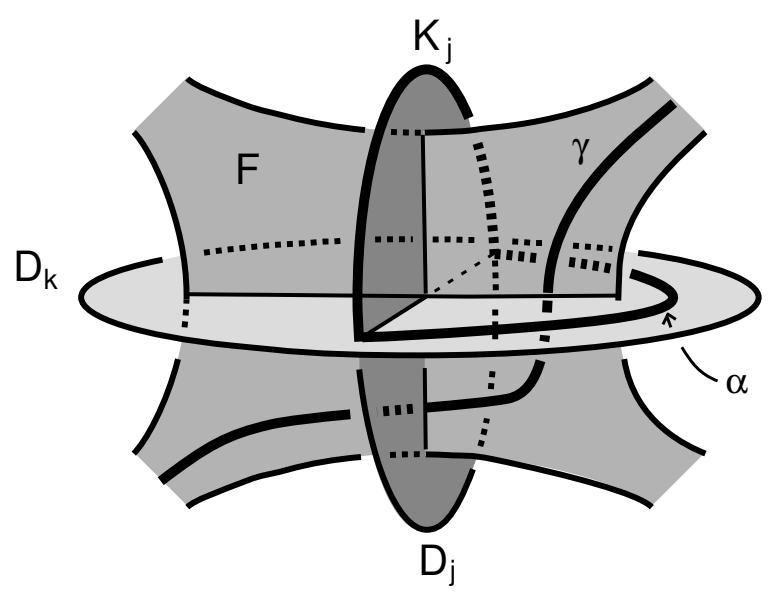

Figure 8.

say above. But from the figure, $\partial \Delta$ has linking number 1 with a loop $\gamma$ in $F$; this loop would therefore have to meet the disk $\Delta$, a contradiction.

\section{The links $L_{n}$ are hyperbolic: Proofs.}

We now verify the four properties needed to show that the links $L_{n}$ are hyperbolic. We work under the assumptions that $n \geq 3$, and the base knot $K$ has $u, v \geq 2$.

Proposition 1. $X\left(L_{n}\right)$ is irreducible: Every embedded 2-sphere bounds a 3-ball.

Proof. Suppose $S$ is a reducing sphere for $X\left(L_{n}\right) . S \subseteq X\left(L_{n}\right) \subseteq S^{3}$, and in $S^{3}, S$ bounds a 3 -ball $B_{1}, B_{2}$ on each side. So we must have $L_{n} \cap B_{i} \neq \emptyset$ for each $i$, otherwise $B_{i} \subseteq X\left(L_{n}\right)$. One of these 3-balls contains $K$, say $B_{1}$.

$F \subseteq X\left(L_{n}\right)$ is incompressible, since a compressing disk for $F$ in $X\left(L_{n}\right)$ would be a compressing disk in $X(K)$. By a standard argument, we can then make $S$ disjoint from $F$ : For any innermost loop of $S \cap F$ in $F$, we can surger $S$ along the corresponding disk in $F$, creating a pair of 2 -spheres, at least one of which must still be a reducing sphere for $X\left(L_{n}\right)$, with fewer circles of intersection with $F$. Therefore, $F \subseteq B_{1}$, since $\partial F=K \subseteq B_{1}$. But each component $K_{i}$ of $L_{n}$ has nonzero linking number with some loop $\gamma$ on $F$ (Figure 8); in particular, $K_{i} \cup \gamma$ is a nonsplit link. But $K_{i} \cup \gamma$ is disjoint from $S$, and so is completely contained in either $B_{1}$ or $B_{2}$. Since $\gamma \subseteq F \subseteq B_{1}$, we have $K_{i} \subseteq B_{1}$ for each $i$. Therefore, $L_{n} \subseteq B_{1}$, and so $L_{n} \cap B_{2}=\emptyset$, a contradiction. So no reducing spheres exist. 
Proposition 2. $X\left(L_{n}\right)$ is $\partial$-irreducible: $\partial X\left(L_{n}\right)$ is incompressible in $X\left(L_{n}\right)$.

Proof. Suppose $D$ is a compressing disk for $\partial X\left(L_{n}\right)$. Since $K$ is a nontrivial knot, $\partial X(K)$ is incompressible in $X(K)$, and so $\partial D$ must lie on $\partial N\left(K_{i}\right)$ for some $i$. It therefore represents a curve of slope $a_{i} / b_{i}$ on $\partial N\left(K_{i}\right)$. Since $F$ is incompressible and disjoint from $N\left(K_{i}\right), D$ and $F$ meet in loops trivial on both, and so we can make $D$ and $F$ disjoint by disk swapping. But then $D$ is disjoint from the loop $\gamma$ of the previous proof, and so $\partial D$ is nullhomologous in the complement of $\gamma$. Since $\partial D$ represents $b_{i}\left[K_{i}\right]=b_{i}$ in $H_{1}(X(\gamma))$, we have $b_{i}=0$, so $\partial D$ is a meridian loop on $\partial N\left(K_{i}\right)$. Capping off with a meridian disk, we get a 2 -sphere in $S^{3}$ meeting $K_{i}$ in a single point, a contradiction. So $D$ does not exist.

Proposition 3. $X\left(L_{n}\right)$ is atoroidal: Every incompressible torus in $X\left(L_{n}\right)$ is $\partial$-parallel.

Proof. Suppose $T$ is an incompressible torus in $X\left(L_{n}\right)$, and suppose, by way of contradiction, that it is not $\partial$-parallel. Since $F$ is incompressible in $X(K)$ and disjoint from $L_{n}$, it is also incompressible in $X\left(L_{n}\right)$, and by a standard disk swapping argument we can make $T \cap F$ consist of loops that are essential on both $T$ and $F$.

Consider $T \cap F \subseteq F$. The loops fall into two types: Those that are parallel to $\partial F$, and those that are not (which are all parallel to one another, however, since $F$ is a once-punctured torus). We begin by showing that we can use $T$ to find a different, essential, torus disjoint from $F$.

Of the $\partial$-parallel loops, the outermost (i.e., $\partial F$-most) loop cobounds an annulus $A$ with $\partial F$, and we can use this annulus to isotope $T$ (in $S^{3}$; in fact, in $\left.X\left(L_{n} \backslash K\right)\right)$ across $\partial F=K$ to a torus $T^{\prime} \subseteq X\left(L_{n}\right)$ (see Figure 9).

Lemma 3. $T^{\prime}$ is incompressible and not $\partial$-parallel in $X\left(L_{n}\right)$.

Proof. If this is not the case, then one of two things is true:

(1) $T^{\prime}$ is $\partial$-parallel.

In this case, if $T^{\prime}$ is parallel to $\partial N(K)$, then the 'dual' annulus $A^{\prime}$ joining $T^{\prime}$ to $\partial N(K)$ (Figure 9) would cut the product region between $T^{\prime}$ and $\partial N(K)$ into a solid torus. By pushing $T^{\prime}$ back across $K$ using $A^{\prime}$, we can then see that our original $T$ bounds a solid torus, a contradiction. But if $T^{\prime}$ is parallel to $\partial N\left(K_{i}\right)$, then $A^{\prime}$ lies outside of the product region, and the annulus $A^{\prime}$ together with an annulus in $\partial N(K)$ and an annulus $A^{\prime \prime}$ in the product region between $T^{\prime}$ and $\partial N\left(K_{i}\right)$ can be stitched together to form an annulus between $\partial N(K)$ and $F$. This annulus might meet $F$ in it's interior, that is, in the interior of $A^{\prime \prime}$. But every loop of intersection will either be trivial in $A^{\prime \prime}$, and so trivial in $F$ by incompressibility, and so can be removed by isotopy of $A^{\prime \prime}$, or is parallel to $\partial A^{\prime \prime}$, and so a $\partial N\left(K_{i}\right)$-most such loop cuts off an annulus between $\partial N(K)$ and $F$, missing $F$ in its interior. In the absence of any such 


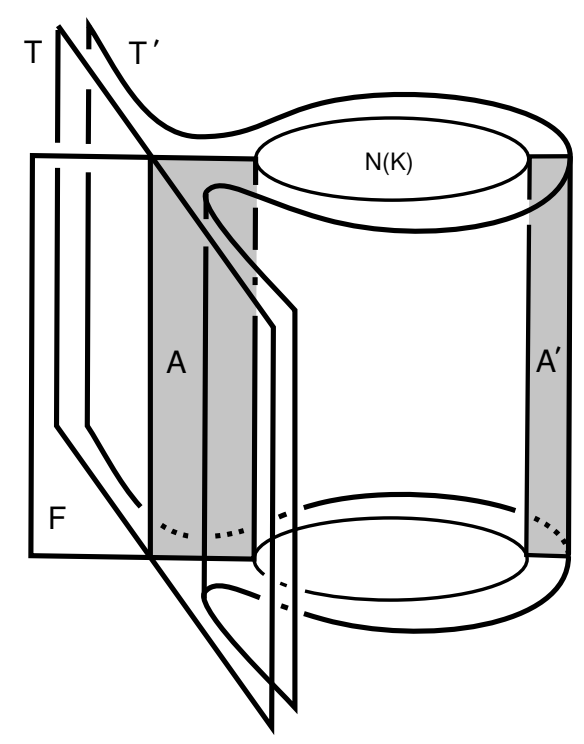

Figure 9.

loops, our original annulus misses $F$ in its interior. But the existence such an annulus will contradict Lemma 1 . So this case cannot occur.

(2) $T^{\prime}$ is compressible in $X\left(L_{n}\right)$, via a compressing disk $D$.

Compressing $T^{\prime}$ along $D$ produces a 2-sphere $S \subseteq X\left(L_{n}\right)$. Since $X\left(L_{n}\right)$ is irreducible by Proposition $1, S$ bounds a 3-ball in $X\left(L_{n}\right)$. This 3-ball either contains $T^{\prime}$, or its interior is disjoint from $T^{\prime}$. Therefore, $T^{\prime}$ either lies in a 3 -ball $B_{0}$ containing $D$, or bounds a solid torus $M_{0}$ containing $D$ (Figure 10). Also, since $T^{\prime} \subseteq X\left(L_{n}\right) \subseteq S^{3}, T^{\prime}$ separates $X\left(L_{n}\right)$.

The dual annulus $A^{\prime}$ is incompressible in $X\left(L_{n}\right) \backslash T^{\prime}$, since $\partial A^{\prime} \cap \partial N(K)$ is an essential loop in $\partial N(K)$, hence in $X\left(L_{n}\right)$. If $D$ and $A^{\prime}$ lie on the same side of $T^{\prime}$, then $D \cap A^{\prime} \subseteq A^{\prime}$ consists of loops and arcs. Since $A^{\prime}$ is incompressible, all of the loops are trivial, and since $D$ is disjoint from $\partial N(K)$, no arc joins the two $\partial$-components of $A^{\prime}$, and so all are $\partial$-parallel. By disk-swapping, we can remove the loops of intersection, and by using the disk cut off by an outermost arc, we can $\partial$-compress $D$ to two disks, at least one of which must still be a compressing disk. After replacing $D$ by one of these disks and continuing, we can eventually find a compressing disk disjoint from $A^{\prime}$, which we will still call $D$.

If $T^{\prime}$ bounds $M_{0}$, then since $M_{0}$ must be disjoint from $\mathrm{K}$ it is also disjoint from the interior of $A^{\prime}$ (Figure 10), i.e., $A^{\prime}$ lies outside of $M_{0}$. The loop $\partial A^{\prime} \cap T^{\prime}$ must represent a generator of $\pi_{1}\left(M_{0}\right)$; otherwise the loop is meridional and so $A^{\prime}$ together with a meridian disk of $M_{0}$ form a compressing 

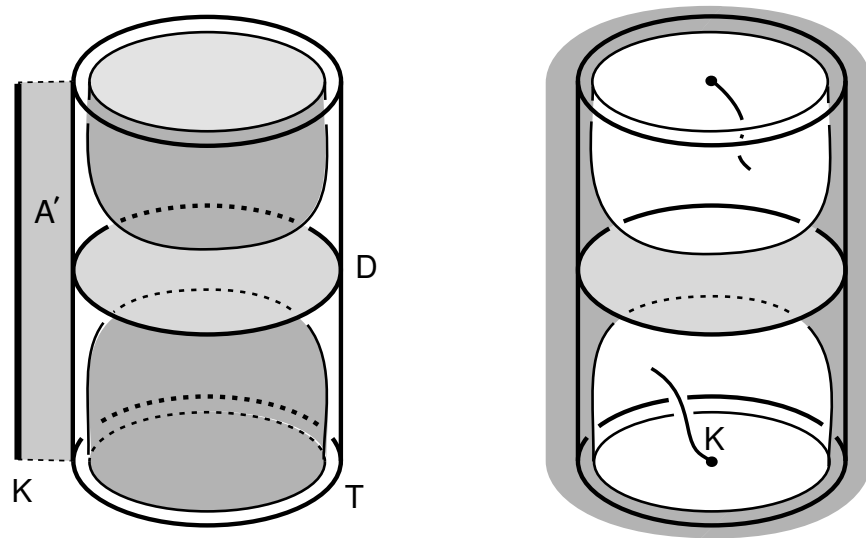

Figure 10.

disk for $\partial N(K)$ in $X\left(L_{n}\right)$, a contradiction, or the loop represents a nontrivial multiple of the generator, and so $A^{\prime}$ represents an isotopy of $K$ to a nontrivial cable of the core of the solid torus $M_{0}$, contradicting the fact $[\mathbf{H T}]$ that, for $|u|,|v| \geq 2$, the 2-bridge knots with continued fraction [2u,2v] are hyperbolic. Therefore, when we push $T^{\prime}$ back to $T$ along $A^{\prime}$, we see that $T$ is parallel to $\partial N(K)$, a contradiction.

If $T^{\prime}$ is contained in a 3-ball $B_{0}$, then since $K$ is disjoint from $B_{0}, D$ and $A^{\prime}$ lie on the same side of $T^{\prime}$, and so we may assume, by the above argument, that they are disjoint. But then when we push $T^{\prime}$ back across $\partial N(K)$ via $A^{\prime}$, the compressing disk $D$ persists, so $T$ is compressible, a contradiction. So this case also cannot occur.

Therefore, pushing $T$ across $A$ to $T^{\prime}$ will always result in another incompressible, non- $\partial$-parallel torus in $X\left(L_{n}\right)$. Continuing for all of the $\partial$-parallel loops of $T \cap F$, we arrive at a new essential torus, which we will still call $T$, having no loops of intersection with $F$ parallel to $\partial F$, i.e., all loops of intersection are nonseparating on $F$. These loops cut $T$ into annuli, and $F$ into annuli and a once-punctured annulus. Since $T$ is separating, none of the annuli in $F$ run from one side of $T$ to the other. One of the boundary components of the once-punctured annulus is the longitude of $\partial N(K)$. Note that $F$ and $T$ cannot meet in a single loop $\gamma$; since such a loop is nonseparating on $F$, a loop in $F$ meeting $\gamma$ in a single point is a loop meeting $T$ in a single point, implying that $T$ is not separating. In fact, this implies that $F$ and $T$ meet in an even number of loops, since otherwise the same loop will meet $T$ in an odd number of points, implying they have nonzero homological intersection number, so $T$ is nontrivial in $H_{2}\left(S^{3}\right)$, a contradiction. $F \backslash T$ therefore consists of an odd number of annuli and a once-punctured annulus. 
Because $X(K)$ is hyperbolic, $T$, thought of as sitting in $X(K)$, must be either compressible or $\partial$-parallel. It cannot be $\partial$-parallel, however, since then the once-punctured annulus component $P$ of $F \mid T$ would have to live in the product region $T \times I$ between $T$ and $\partial N(K)$. $P$ must therefore be compressible in $T \times I$ (since its fundamental group, being nonabelian, cannot inject), implying that $F$ is compressible in $X(K)$, a contradiction.

We also know that since $T \subseteq X\left(L_{n}\right) \subseteq X(K) \subseteq S^{3}$, T bounds a solid torus $M_{0}$ in $S^{3}$. In fact, $T$ must either bound a solid torus in $X(K)$, or $K$ must lie in a 3 -ball in a solid torus with boundary $T$. For if not, then $T$ cannot bound a solid torus on both sides ( $K$ would be disjoint from one of them). $T$ is therefore the boundary of a neighborhood of a nontrivial knot, and so is incompressible on the side away from $M_{0}$. Therefore $K$ lies in $M_{0}$ and $T$ is incompressible on the side away from $K$. But $K$ is not isotopic in $M_{0}$ to the core $C$ of $M_{0}$, since $T$ is not $\partial$-parallel in $X(K)$, and $C$ is a nontrivial knot (since $T$ is the incompressible boundary of $X(C)$ ). So either $T$ is incompressible in $X(K) \cap M_{0}=M_{0} \backslash \operatorname{int} N(K)$, so $K$ is a satellite knot (and therefore not hyperbolic, a contradiction), or $T$ is compressible in $X(K) \cap M_{0}$, and so $K$ misses a meridian disk for $M_{0}$ (the only compressing disk we could have). So either $M_{0} \subseteq X(K)$, or $K \subseteq M_{0}$ and misses a meridian disk for $M_{0}$.

If $M_{0} \subseteq X(K)$ and $T \cap F \neq \emptyset$, then there must be at least one annulus of $F \backslash T$ in $M_{0}$, so there exists an outermost such annulus $A$. If we split $T$ open along $A$ and glue two parallel copies of $A$ onto the resulting annuli, we obtain two new tori $T_{1}$ and $T_{2}$ in $X\left(L_{n}\right)$, with $T_{1}$ (say) disjoint from $F$, each bounding a solid sub-torus in $X(K)$ (see Figure 11), and joined to $F$ by 'dual' annuli $A_{1}$ and $A_{2}$. Neither of these tori can be $\partial$-parallel in $X\left(L_{n}\right)$, otherwise the argument of Lemma 3 will find an annulus contradicting Lemma 1. If $T_{1}$ is compressible, by a compressing disk $D_{1}$, then since $T_{1}$ is disjoint from $F$ we can by the usual disk-swapping process make $D_{1}$ disjoint from $F$ as well.

But then, as in Lemma 3, either $T_{1}$ bounds a solid torus $M_{1}$ in $X\left(L_{n}\right)$, and $A_{1}$ lies on the opposite side of $T_{1}$ (since $F$ does), or $T_{1}$ lies in a 3 -ball $B_{1}$, and $D_{1}$ and $A_{1}$ lie on the same side of $T_{1}$. In the first case, this solid torus must then be identical to the one we see inside of $M_{0}$ in Figure 11, and so we can use this solid torus to isotope the annulus of $T \backslash F$ in $T_{1}$ across $F$, reducing the number of components of $T \cap F$. In the second case, if $D_{1}$ and $A_{1}$ lie on the same side of $T_{1}$, then $D_{1} \cap A_{1} \subseteq A_{1}$ does not contain an essential loop, since otherwise the sub-annulus cut off in $A_{1}$ parallel to $F$ together with the the subdisk cut off in $D_{1}$ would give a (singular) compressing disk for $F$, a contradiction. Therefore by the same process used in the proof of Lemma 3 , we can make $D_{1}$ disjoint from $A_{1}$ as well. But then $D_{1}, A_{1}$, and an annulus in $T_{1}$ between their $\partial$-components together form a compressing 


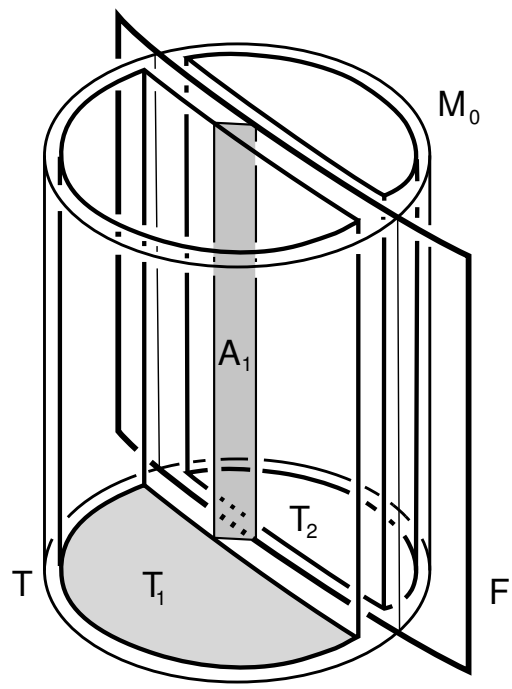

Figure 11.

disk for $F$, a contradiction. Therefore, when $M_{0} \subseteq X(K)$, we can always reduce the number of components of $T \cap F$.

If $K \subseteq M_{0}$ and misses a meridian disk $D$ for $M_{0}$, and $T \cap F \neq \emptyset$, then by the incompressibility of $F$ and the irreducibility of $X(K)$ we can isotope $D$ rel $\partial D$ to remove any circles of intersection with $F$. $D \cap F$ must still then be nonempty, since otherwise $D$ together with an annulus in $T$ between $\partial D$ and a component of $T \cap F$ gives a compressing disk (in $X(K)$ ) for $F$. In particular, the loops of $T \cap F$ are not meridians for $M_{0}$; otherwise, by isotopy we could make $\partial D$ (and therefore $D$ ) disjoint from $F$. By an isotopy of $F$ in $X\left(L_{n}\right)$, we can assume that these loops meet $\partial D$ minimally.

If $T \cap F$ has only two components, then $F \cap M_{0}$ consists of a oncepunctured annulus $P$, with two $\partial$-components on $\partial M_{0}$, and the other $\partial$ component equal to $K$. Loops of $P \cap D \subseteq D$ can be removed by disk swapping, since $P \subseteq F$ is incompressible in $X(K) \cap M_{0} ; P \cap \partial M_{0}$ consists of loops essential in $F$. Then $P \cap D$ consists of arcs; an outermost such arc $\alpha$ must join distinct $\partial$-components of $P$ together, since $T \backslash P$ consists of a pair of annuli, each of whose $\partial$-components come from distinct components of $\partial P$. But then the outermost disk this arc cuts off, together with the annulus in $T$, can be used to build a disk $D^{\prime}$ with $\partial D^{\prime} \subseteq F$ (Figure 12). Since $F$ is incompressible, $\partial D^{\prime}$ bounds a disk in $F$. But since $\alpha$ joins distinct components of $\partial P, \alpha$ cuts $P$ into an annulus, one of whose $\partial$-components is $\partial D^{\prime}$, implying that $F$ is a disk, a contradiction. So $T \cap F$ must have more than two components. 


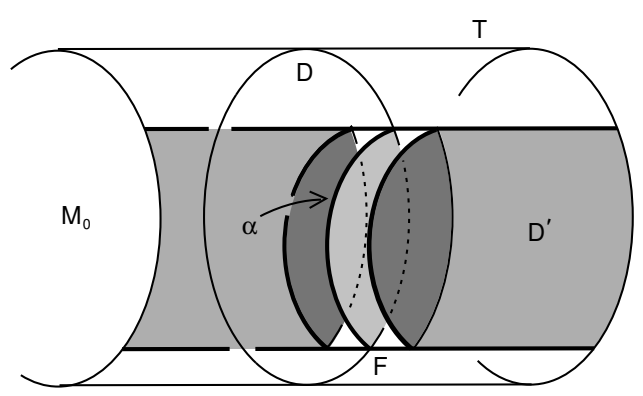

Figure 12.

If $T \cap F$ consists of more than two components, then there is at least one annulus component of $F \cap M_{0}$. An outermost such annulus cuts a solid torus $M_{1}$ off from $M_{0}$. If $M_{1}$ does not contain $K$, then we can apply the argument given above for the case that $M_{0} \subseteq X(K)$ to show that we can reduce the number of components of $T \cap F$ by an isotopy of $T$. If $M_{1}$ does contain $K$, then it also contains $P$, and so we have a situation identical to the one in the previous two paragraphs; the meridian disk for $M_{1}$ is a sub-disk of $D$, and so misses $K$. This will lead us to the same contradiction.

Therefore, we can either replace $T$ with an essential torus $T_{1}$ disjoint from $F$, or reduce the number of components of $T \cap F$ by an isotopy of $T$ in $X\left(L_{n}\right)$. Eventually, therefore, we can find an essential torus $T$ with $T \cap F=\emptyset$.

Once we have found an essential torus $T$ with $T \cap F=\emptyset$, we turn our attention to $T \cap D_{1}$, where $D_{1}$ is the disk of Section 1 bounding $K_{1} \subseteq$ $L_{n}$. Since $T$ is disjoint from $F$ and $K_{1}$, after removing trivial circles of intersection in $D_{1}$, which are therefore trivial on $T$, as well, $T \cap D_{1}$ consists of loops which miss the arc $F \cap D_{1}$, so all of the loops are parallel, in $D_{1}$, to $\partial D_{1}=K_{1}$. The outermost such loop cuts off an annulus $A_{1}$ from $D_{1}$ (Figure 13), which we use as in Lemma 3 to push $T$ across $K_{1}$ to a new torus $T^{\prime}$ in $X\left(L_{n}\right)$.

Lemma 4. $T^{\prime}$ is incompressible and not $\partial$-parallel in $X\left(L_{n}\right)$.

Proof. Most of our arguments follow the same line as the proof of Lemma 3. If $T^{\prime}$ is parallel to $\partial N\left(K_{i}\right)$, then if $i \neq 1$, we can use the dual annulus $A_{1}^{\prime}$ from $T^{\prime}$ to $\partial K_{1}$ together with an annulus in the product region to build an annulus in $X\left(L_{n}\right)$ between $\partial N\left(K_{1}\right)$ and $\partial N\left(K_{i}\right)$, contradicting Lemma 2. If $T^{\prime}$ is parallel to $\partial N(K)$, then $A_{1}^{\prime}$ together with an annulus in the product region and an annulus in $\partial N(K)$ gives an annulus in $X\left(L_{n}\right)$ between $\partial X\left(K_{1}\right)$ and $F$, contradicting Lemma 1 . And if $T^{\prime}$ is parallel to $\partial N\left(K_{1}\right)$, then the dual annulus $A_{1}^{\prime}$ splits the product region into a solid torus; pushing $T^{\prime}$ back 


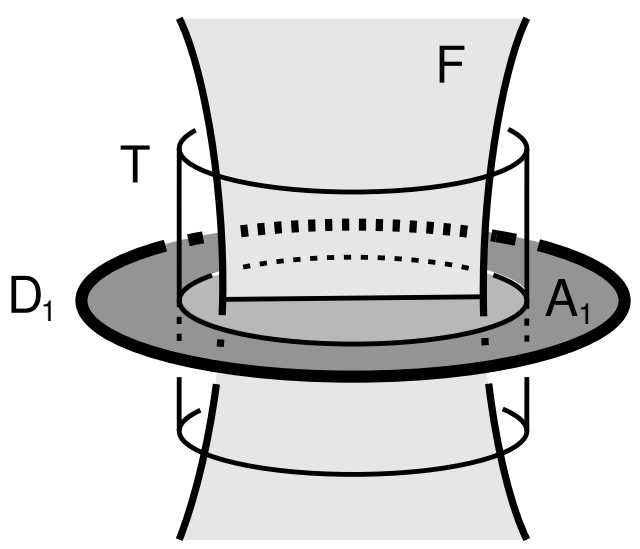

Figure 13.

to $T$ along $A_{1}^{\prime}$ essentially preserves this solid torus, implying that $T$ bounds a solid torus in $X\left(L_{n}\right)$, a contradiction. So $T^{\prime}$ is not $\partial$-parallel.

If $T^{\prime}$ is compressible, then either $T^{\prime}$ bounds a solid torus $M_{1}$, or $T^{\prime}$ is contained in a 3-ball $B_{1}$ in $X\left(L_{n}\right)$. If $T^{\prime}$ bounds $M_{1}$, then as before $M_{1}$ is disjoint from the interior of $A_{1}^{\prime}$. Note also that $M_{1}$ is disjoint from $F$, since $T \cap F=\emptyset$ and $M_{1}$ does not meet $\partial F=K$. As before, $\partial A_{1}^{\prime} \cap M_{0}=\gamma_{1}$ must represent a generator of $\pi_{1}\left(M_{1}\right)$. Otherwise, either $\gamma$ is a meridian $M_{1}$, and a meridian disk together with $A_{1}^{\prime}$ gives a disk with boundary $K_{1}$ disjoint from $F$, implying that $K_{1}$ has linking number zero with every loop in $F$, a contradiction, or $\gamma_{1}$ represents a nontrivial multiple of the core $C$ of $M_{1}$, so $\gamma_{1}$, and therefore $K_{1}$, is homologous to a multiple $r$ of $C$ in the complement of $F$, implying that $K_{1}$ has linking number a multiple of $r$ with every loop in $F$, contradicting the fact that it has linking number one with some loops (e.g., the $\gamma$ of Figure 8 above). But now when we push $T^{\prime}$ back to $T$ using $A_{1}^{\prime}, K_{1}$ is pushed to the core of the solid torus $M_{1}$, implying that $T$ is $\partial$-parallel, a contradiction.

Finally, if $T^{\prime}$ and its compressing disk $D$ lie in a 3-ball $B_{1}$ (the only remaining possibility), then $D$ and $A_{1}^{\prime}$ lie on the same side of $T^{\prime}$, and so, as in Lemma 3, we can make $D$ disjoint from $A_{1}^{\prime}$. Then when we push $T^{\prime}$ back to $T$ across $A_{1}^{\prime}$, the compressing disk persists, so $T$ is compressible, a contradiction. So $T^{\prime}$ must be incompressible and not $\partial$-parallel.

We can apply this argument to each loop of $T \cap D_{1}$ in turn, pushing them across $K_{1}$ to obtain a new essential torus. After carrying this out for all loops of intersection, we can then assume that $T \cap F$ and $T \cap D_{1}$ are both empty. We then turn our attention to $T \cap D_{2}$, which as before consists of loops in $D_{2}$ parallel to $\partial D_{2}=K_{2}$. By the same process as in 
Lemma 4, we remove these loops of intersection as well. Continuing, we eventually find an essential torus $T$ which is disjoint from $F$ and all of the disks $D_{i}, i=1, \ldots, n$. $T$ therefore lives in $S^{3} \backslash \operatorname{int}\left(F \cup D_{1} \cup \cdots \cup D_{n}\right)$, which as we remarked in Section 2, is a handlebody $H$. But every torus in a handlebody is compressible $\left(\pi_{1}(T)\right.$ is not free, so it cannot inject into $\left.\pi_{1}(H)\right)$. This compressing disk misses $F$ and all of the $D_{i}$, and so it lives in $X\left(L_{n}\right)$. Therefore $T$ is compressible in $X\left(L_{n}\right)$, a contradiction. So no such (original) torus can exist; $X\left(L_{n}\right)$ is atoroidal.

Proposition 4. For $n \geq 3, X\left(L_{n}\right)$ is anannular: Every incompressible annulus is d-parallel.

Proof. The argument here is standard, we simply use the facts that $X\left(L_{n}\right)$ is irreducible and atoroidal, and has at least four $\partial$-components (since $n \geq 3$ ).

If $A$ is an incompressible annulus, then if $A$ runs between distinct $\partial$ components $T_{1}, T_{2}$, then $T=\partial N\left(A \cup T_{1} \cup T_{2}\right) \backslash\left(T_{1} \cup T_{2}\right)$ is a torus which separates pairs of $\partial$-components of $X\left(L_{n}\right)$, so cannot be $\partial$-parallel, and must therefore be compressible. But a compressing disk will split $T$ into a 2-sphere which also separates components of $L_{n}$, implying that $X\left(L_{n}\right)$ is reducible, a contradiction. So $\partial A$ is contained in a single $\partial$-component $T_{1}$. Then $T=\partial N\left(A \cup T_{1}\right) \backslash T_{1}$ is a torus which separates $T_{1}$ from at least three other $\partial$-components. So if $T$ is $\partial$-parallel, it is parallel to $T_{1}$, so $A$ lives in a product $T \times I$, and so is $\partial$-parallel. If $T$ is compressible, then the compressing disk splits $T$ into a 2 -sphere separating $T_{1}$ from at least three other $\partial$-components, giving a reducing sphere for $X\left(L_{n}\right)$, a contradiction.

The only possibility which does not lead to a contradiction, therefore, is that $A$ is $\partial$-parallel. Therefore, $X\left(L_{n}\right)$ is anannular.

\section{Concluding remarks.}

With this we have finished our proof that the links $L_{n}$ are hyperbolic. By applying the construction of Section 1, we can therefore build infinitely many (distinct) knots with free genus one and volume larger than any fixed constant. We find it both amusing and embarrassing to note that we can, however, not exhibit a single explicit example of this phenomenon, for any fixed constant. Existence of our examples is guaranteed only by Thurston's hyperbolic Dehn surgery theorem, which provides no explicit estimate the sizes of coefficients $n_{i}$ sufficient to guarantee hyperbolicity of the knots we build. And while there are estimates of the volume of a hyperbolic manifold after Dehn filling (see, e.g., [NZ]), these are asymptotic estimates, giving no explicit lower bounds in terms of the $n_{i}$.

It is clear that similar constructions can be carried out starting with free genus one knots other than the $[2 u, 2 v]$ 2-bridge knots. All that is really needed to carry over our proofs is an analogue to Lemma 1 . Our interest here was in finding hyperbolic knots with free genus one; we should note that 


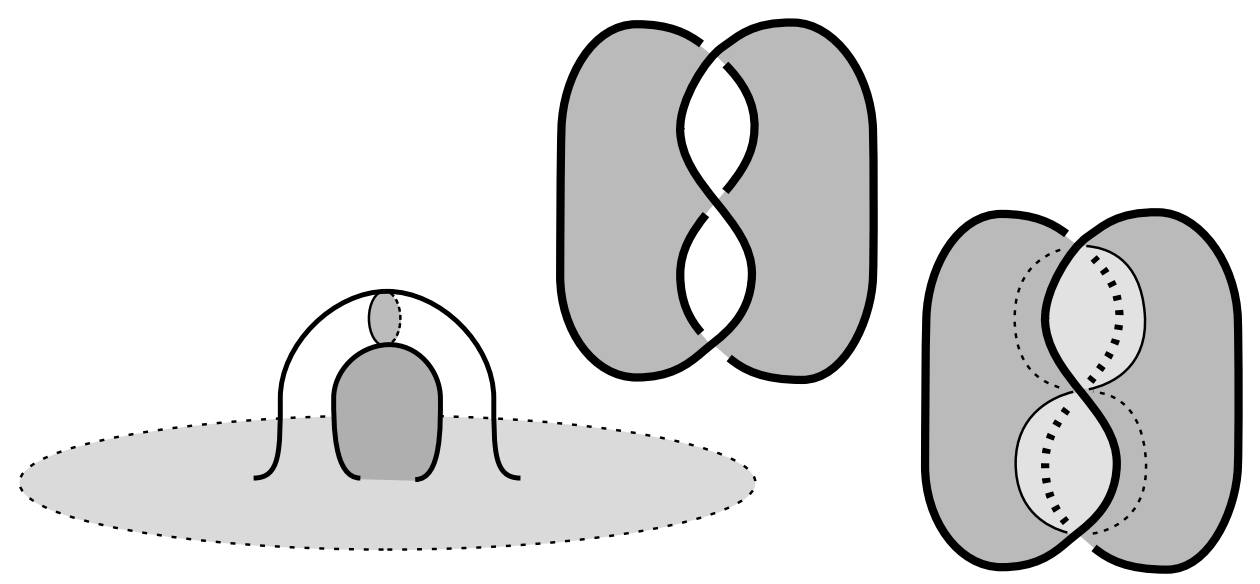

Figure 14.

Ozawa $[\mathbf{O z}]$ has, on the other hand, determined all of the satellite knots with free genus one. In that paper, he conjectures that any free genus one knot whose exterior contains an essential closed surface must also have tunnel number one. His paper essentially consists of a proof of this conjecture when the exterior contains an essential torus. This, together with a result of Goda and Teragaito [GT], gives the required characterization.

A free Seifert surface remains free after stabilization, i.e., after adding a trivial 1-handle the surface (see Figure 14). It is easy to see that a canonical surface stabilizes to a canonical one; stabilization can be thought of as boundary connected sum with a canonical surface for the unknot (Figure 14), and the connected sum of a diagram for $K$ and a diagram for the unknot is a diagram for $K$. It would be interesting to determine whether or not any two free Seifert surfaces are stably equivalent, i.e., they become isotopic after a sufficient number of stabilizations. (Since the effect, on the complement, of a stabilization is to boundary-connect sum with two solid tori, you need to start with handlebody complement in order to get handlebody complement.) This is probably not unreasonable, since this operation is very similar to the stabilization of Heegaard decompositions (see, e.g., $[\mathbf{A M}]$ ), where stable equivalence is known.

This stabilization process raises several further interesting possibilities. Can every free Seifert surface be stabilized to a canonical one? In particular, might it be the case that any free Seifert surface, if it is stabilized to have genus equal to (or greater than) the canonical genus, must then always be canonical? This would imply that if the free genus equals the canonical genus, then every free Seifert surface is canonical. The standard conjecture in the theory of Heegaard decompositions (see, e.g., [Ki, Problem 3.89]) seems to be that for any pair of Heegaard decompositions of a 3-manifold, 
they are equivalent after stabilizing to a genus one higher than the larger of the two. Perhaps a similar result is true for free Seifert surfaces, as well.

Acknowledgements. The author wishes to thank Jeff Weeks for many helpful conversations during the course of this work, and Tsuyoshi Kobayashi and Nara Women's University for providing the setting in which these conversations took place. The author also wishes to thank Susan Hermiller for help in deciphering normal forms in free groups.

\section{References}

[Ad] C. Adams, Volumes of $N$-cusped hyperbolic 3-manifolds, J. London Math. Soc., 38 (1988), 555-565, MR 89k:22020, Zbl 627.57013.

[AM] S. Akbulut and J. McCarthy, Casson's invariant for oriented homology 3-spheres, Mathematical Notes, 36, Princeton University Press, 1990, MR 90k:57017, Zbl 695.57011.

[Br] M. Brittenham, Bounding canonical genus bounds volume, preprint.

[GT] H. Goda and M. Teragaito, Tunnel number one genus one non-simple knots, Tokyo J. Math., 22 (1999), 99-103, MR 2000j:57011, Zbl 939.57009.

[HT] A. Hatcher and W. Thurston, Incompressible surfaces in 2-bridge knot complements, Inventiones Math., 79 (1985), 225-246, MR 86g:57003, Zbl 602.57002.

[Ki] R. Kirby, Problems in low-dimensional topology, in 'Geometric topology' (Athens, GA, 1993), Studies in Advanced Mathematics, 2, Amer. Math. Soc., Providence, RI, (1997), 35-473, CMP 1470751.

[KK] M. Kobayashi and T. Kobayashi, On canonical genus and free genus of a knot, J. Knot Thy. Ram., 5 (1996), 77-85, MR 97d:57008, Zbl 859.57009.

[MKS] W. Magnus, A. Karrass and D. Solitar, Combinatorial Group Theory, John Wiley and Sons, Inc., 1966, MR 34 \#7617, Zbl 138.25604.

[NZ] W. Neumann and D. Zagier, Volumes of hyperbolic three-manifolds, Topology, 24 (1985), 307-332, MR 87j:57008, Zbl 589.57015.

[Oz] M. Ozawa, Satellite knots of free genus one, J. Knot Thy. Ram., 8 (1999), 27-31, MR 2000b:57012, Zbl 941.57010.

[Ro] D. Rolfsen, Knots and Links, Publish or Perish Press, 1976, MR 58 \#24236, Zbl 339.55004.

[Se] H. Seifert, Über das Geschlecht von Knoten, Math Annalen, 110 (1934), 571-592.

[Th1] W. Thurston, The Geometry and Topology of 3-manifolds, Notes from lectures at Princeton University, 1978-80.

[Th2] _ Three-dimensional manifolds, Kleinian groups and hyperbolic geometry, Bull. Amer. Math. Soc., 6 (1982), 357-381, MR 83h:57019, Zbl 496.57005.

[We] J. Weeks, SnapPea, a program for creating and studying hyperbolic 3-manifolds, available for download from www.geom.umn.edu.

Received June 24, 1999. This research was supported in part by NSF grant \#DMS-9704811. 
Department of Mathematics

UNIVERSity of NEBRASKA

LinCOLN, NE 68588-0323

E-mail address: mbritten@math.unl.edu 